\title{
Multidisciplinary team approach on a case of bilateral tension pneumothorax
}

\author{
Xiaodong $\mathrm{Li}^{1}$, Xiaofen $\mathrm{Su}^{2}$, Baofu Chen ${ }^{1,3}$, Haijun Yao ${ }^{4}$, Yuetian $\mathrm{Yu}^{5}$, Xuefeng Leng ${ }^{6}$, Qiang $\mathrm{Lu}^{7}$, \\ Chunguang Wang ${ }^{8}$, Juanjuan Lei ${ }^{9}$, Kurt Ruetzler ${ }^{10}$, Hiran C. Fernando ${ }^{11}$, Sebastien Gilbert ${ }^{12,13}$, Yeung \\ Ching $^{12,13}$, Pier Luigi Filosso ${ }^{14}$, Jianfei Shen ${ }^{1}$, Chengchu Zhu ${ }^{1}$; Written on behalf of AME Thoracic \\ Surgery Collaborative Group
}

\begin{abstract}
${ }^{1}$ Department of Cardiothoracic Surgery, Taizhou Hospital of Zhejiang Province, Wenzhou Medical University, Linhai 318000, China; ${ }^{2}$ Department of Respiratory Medicine, the First Affiliated Hospital of Guangzhou Medical University, Guangzhou 510230, China; ${ }^{3}$ School of Medicine, Shandong University, Jinan 250100, China; ${ }^{4}$ Intensive Care Unit, the Sixth People's Hospital of Shanghai Jiao Tong University, Shanghai 200233, China; ${ }^{5}$ Intensive Care Unit, Renji Hospital Affiliated to Shanghai Jiao Tong University School of Medicine, Shanghai 200233, China; ${ }^{6}$ Department of Thoracic Surgery, the Affiliated Hospital of Chengdu University, Chengdu 610081, China; ${ }^{7}$ Department of Thoracic Surgery, Tangdu Hospital, the Fourth Military Medical University, Xi'an 710032, China; ${ }^{8}$ Department of Anesthesiology, Baoding First Central Hospital, Baoding 071028, China; ${ }^{9}$ Department of Rehabilitation, Tangdu Hospital, the Fourth Military Medical University, Xi'an 710032 , China; ${ }^{10}$ Departments of Outcomes Research and General Anesthesiology, Cleveland Clinic, Main Campus, Cleveland, OH, USA; ${ }^{11}$ Inova Schar Cancer Institute, Inova Fairfax Medical Campus, Section of Thoracic Surgery, Department of Surgery 3300 Gallows Road, Falls Church, VA, USA; ${ }^{12}$ Division of Thoracic Surgery, Department of Surgery, Faculty of Medicine, University of Ottawa, Ottawa, Canada; ${ }^{13}$ Division of Thoracic Surgery, Department of Surgery, The Ottawa Hospital, Ottawa, Canada; ${ }^{14}$ Department of Surgical Sciences, University of Torino, Torino, Italy

Correspondence to: Jianfei Shen; Chengchu Zhu. Taizhou Hospital of Zhejiang Province, Wenzhou Medical University, Linhai 318000 , Zhejiang, China. Email: jianfei051@163.com; zhucc@enzemed.com.
\end{abstract}

Submitted Feb 28, 2018. Accepted for publication Apr 10, 2018.

doi: $10.21037 /$ jtd.2018.04.81

View this article at: http://dx.doi.org/10.21037/jtd.2018.04.81

\section{Case presentation}

A 63-year-old male patient was admitted to a local hospital with a four day history of bilateral chest tightness and pain without any recognizable cause. The symptoms worsened after physical activities, along with shortness of breath. At hospital admission, bilateral tension pneumothorax was diagnosed and emergency bilateral closed-chest drainage was performed; however, the symptoms did not improve. Patient was then he transferred to the Department of Cardiothoracic Surgery of Taizhou Hospital of Zhejiang Province for further evaluation and treatment.

The patient had a history of chronic obstructive pulmonary disease (COPD) for 3 years but had no history of hypertension, coronary artery disease, or tuberculosis. The patient was non-smoker and did not consume alcohol. He did not work as pastor or miner and he was not exposed to any chemical substance. During physical examination, the pulse rate was 105 beats per minute, body temperature was $36.5^{\circ} \mathrm{C}$, the respiratory rate (RR) was 25 breaths per minute, blood pressure 139/87 $\mathrm{mmHg}$, body height $172 \mathrm{~cm}$, and body weight was $46 \mathrm{~kg}$. Orthopnea was diagnosed. Crepitus was palpable over his limbs, neck, chest wall, and scrotum. Tympany was heard during percussion in both lungs. Respiratory breath sounds were decreased, while no obvious dry or moist rales were heard. The cardiac rhythm was regular, and no obvious pathological murmur was noticed. Computer tomography (CT) at admission revealed bilateral pneumothorax, massive air accumulation in the subcutaneous tissues of the neck, mediastinum, chest wall/abdominal and pelvic walls, and both scrotums, minor bilateral pleural effusion, and patchy opacities in both lungs (Figure 1). Routine blood testing was performed: white blood cells (WBC), 8.2×109/L; hemoglobin (Hg), $131 \mathrm{~g} / \mathrm{L}$; neutrophil granulocyte ratio (N\%), 92.2\%; and absolute neutrophil count (ANC), 7.6×109/L. Arterial blood gas was analyzed: $\mathrm{pH}, 7.39 ; \mathrm{PO}_{2}, 88 \mathrm{mmHg}$; and $\mathrm{PCO}_{2}, 44 \mathrm{mmHg}$.

After admission to the hospital, the both chest tubes were placed under $2-5 \mathrm{kPa}$ suction. At rest, the patient had an obvious air leak from both chest tubes. Cefoxitin combined with levofloxacin was given IV for antimicrobial 

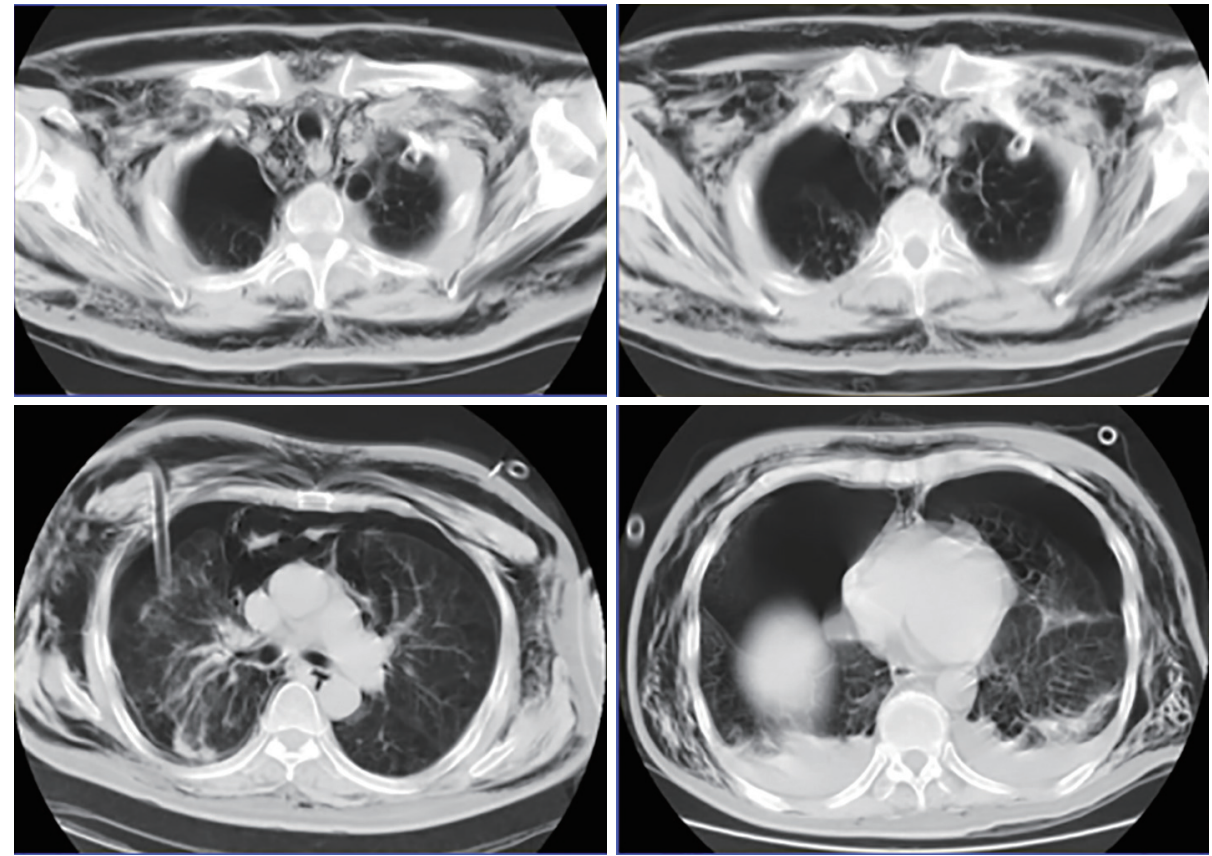

Figure 1 Chest computed tomography (CT) images on admission bilateral pneumothorax (drainage tube indwelling) and massive air accumulation in the subcutaneous tissues of the neck, chest, abdomen, scrotums, and limbs.

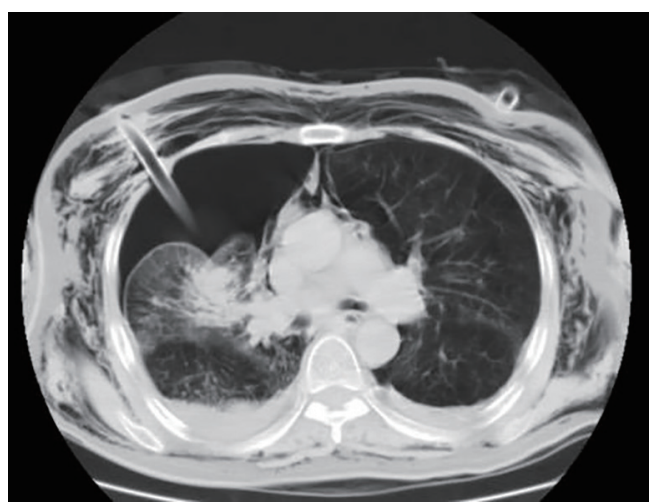

Figure 2 One month after conservative treatment, a second chest CT revealed bilateral pneumothorax (drainage tube indwelling), massive air accumulation in the subcutaneous tissues of the neck, mediastinum, chest wall/pelvic wall, and both scrotums, and a small amount of pleural effusion on both sides. CT, computed tomography.

treatment for 3 days. However, the patient's symptoms did not improve, and the WBC tended up to high up. Consequently, imipenem/cilastatin IV was given.

Other treatments included aminophylline for dilating bronchus and ambroxol hydrochloride for reducing phlegm.
The symptoms of chest tightness were obvious, along with high tension of subcutaneous emphysema, especially on the right chest wall. Also, the patient was found to be coughing up yellow sputum. On the second day after admission, infraclavicular skin incisions (blow holes) were fashioned to release subcutaneous emphysema and the symptoms were slightly alleviated. As symptoms persisted a second closed drainage of the right thoracic cavity was performed at the sixth days after admission. A large amount of air was drained off via three thoracic tubes after the procedure, and the symptoms were relieved. However, one month after the conservative treatment, there was a persistent air leak, and the subcutaneous emphysema was still evident. A second chest CT revealed bilateral pneumothorax (drainage tube indwelling), extensive accumulation of air in the subcutaneous tissues of the neck, mediastinum, chest wall/ pelvic wall, and both scrotums, patchy opacities in both lungs, and minor bilateral pleural effusions (Figure 2). A subsequent arterial blood gas analysis showed that the $\mathrm{PO}_{2}$ was 72 [80-100] $\mathrm{mmHg}$ and the $\mathrm{PCO}_{2}$ was 51 [35-45] $\mathrm{mmHg}$.

\section{Clinical diagnosis}

Bilateral tension pneumothorax, rupture of bilateral 
pulmonary bullae, COPD, massive bilateral subcutaneous emphysema, small amount of pleural effusion on both sides, and pulmonary infections.

\section{Multidisciplinary team (MDT) approach}

The patient was admitted due to "chest pain and tightness at both sides for 4 days" and diagnosed with bilateral tension pneumothorax. After closed-chest drainage on both sides, air leak persisted and subcutaneous emphysema progressively aggravated, along with lung infections. The skin was incised to relieve the subcutaneous air, and additional therapeutic approaches including closed-chest drainage tubes, antibiotics, and auxiliary expectoration were applied. However, the efficacy was poor after 1 month of treatment, and further treatments were discussed.

\section{Inputs from department of respiratory medicine}

Dr. Xiaofen Su (from the First Affiliated Hospital of Guangzhou Medical University): The patient had a poor nutritional status, including wasting. His body mass index (BMI) was only 15.5. He had a history of COPD. The bilateral pneumothorax seen in him was a secondary condition. Conservative treatment should be the primary choice. Active infection control, control of COPD symptoms, and medical conservative treatment can be applied. Surgical treatment might be considered if these interventions failed.

If the conservative treatment continues, the following measures may be taken: (I) serological and pathogenic specimens are taken to evaluate the infection, and antimicrobial treatment is actively performed; (II) the patient has COPD, with hypoxia and carbon dioxide retention. Thus, measures (e.g., relieving cough, eliminating phlegm, relieving spasm, and alleviating asthma) for enhancing clearance of airway secretions may be applied to keep the airway patent. In necessary, noninvasive ventilation may be used to improve ventilation and oxygenation, which needs to be performed under closed-chest drainage; (III) since the patient had bilateral pneumothorax, persistent air leak, and systemic subcutaneous emphysema, mediastinotomy for continuous drainage may be performed under a negative pressure of $1-2 \mathrm{kPa}$ during the bilateral closed-chest drainage. If the air leak persists (i.e., bubbles continue to emit from the water-sealed drainage bottle 24-48 hous later), pleural adhesion therapy may be applied when both lungs are almost completely recruited. If the lungs are still not recruited 5 days later or if the rupture is still not closed after treatment for pleural adhesions, surgical treatment may be considered.

If surgery is required, the patient should be transferred to intensive care unit (ICU) immediately after the procedure. Suggestions on respiratory management: invasive ventilator ventilation is performed under tracheal intubation, which facilitates airway management, ensures airway patency and drainage of sputum, improves ventilation and oxygenation, and relieve carbon dioxide retention. The tubes may be withdrawn after the condition becomes stable. Other treatments: measures for relieving cough, eliminating phlegm, relieving spasm, and alleviating asthma continue; in addition, early enteral nutrition ensures nutritional support and protein delivery and promotes wound healing.

\section{Inputs from ICU}

Dr. Haijun Yao (from the Intensive Care Unit, the Sixth People's Hospital of Shanghai Fiao Tong University): Based on the symptoms, signs, and chest CT findings of the patient, a definite diagnosis of pneumothorax can be made. After a long period of conservative treatment, the symptoms have not been well relieved and the patient still suffers from pulmonary air leaks and atelectasis, with manifestations of increased lung infection and respiratory failure. Thus, surgery should be the first choice.

If the patient refuses surgical treatment, F16 or even smaller chest tubes may be placed for closed-chest drainage, and suctioning with low negative pressure (suction force $<3 \mathrm{kPa}$ ) continues. The secretions, blood samples, and sputum samples were cultured and drug susceptibility testing was performed. The antimicrobial use is adjusted according to drug susceptibility testing results.

If surgical treatment is performed, staged surgery should be applied for both sides of pneumothorax according to the patient's current condition. The right side can be surgically treated firstly because the right side is relatively large; if there is any problem, the left side may still be compensated. The specific operation protocol (repair or resection of parts of lung tissues) needs to be determined by thoracic surgeons based on the intraoperative histopathology. After the operation on the right side is completed, an operation on the left side may be considered after the condition becomes stable. The specific surgical protocol may be same as that on the right side.

Double-lumen tracheal intubation under general 
anesthesia should be applied, during which attention should be paid to the location of the endotracheal tube and the vital signs and the end-tidal carbon dioxide partial pressure should be closely monitored. If the oxygen partial pressure cannot be properly maintained, intermittent two-lung ventilation may be applied.

The principles of postoperative management include: (I) during the ventilator support after the surgery, the use of positive end-expiratory pressure (PEEP) should be minimized; (II) clinically satisfactory oxygen saturation (i.e., 92\%) should be achieved with the lowest oxygen concentration; (III) the endotracheal tube should be removed as early as possible, along with atomization treatment, reducing phlegm, and other symptomatic measures; (IV) if the oxygen saturation decreases after extubation, oxygen inhalation mask or noninvasive ventilator-assisted high-volume ventilation (high flow) may be considered; (V) the patients should be encouraged to be ambulatory as early as possible; (VI) postoperative nutritional support should be strengthened; and (VII) the air inflow and outflow should be strictly controlled to reduce pulmonary interstitial effusion and improve the ventilation/perfusion ratio.

Dr. Yuetian Yu (from Renji Hospital, Shanghai fiaotong University School of Medicine): The patient was initially diagnosed with tension pneumothorax. He received bilateral closed-chest drainage, followed by the use of a second-generation cephalosporin (cefuroxime $1.5 \mathrm{~g}$ IV $\mathrm{q} 12 \mathrm{~h}$ ), which met the guidelines on antimicrobial therapy. The patient suffered from cough with yellow viscous sputum after cardiothoracic surgery. Since he also had new exudates on both lower lungs, hospital-acquired pneumonia is considered. The patient had a history of COPD. After hospital-acquired pneumonia occurs, sputum culture should be performed to isolate the pathogen, along with antimicrobial susceptibility test. Since the secondgeneration cephalosporin was ineffective, levofloxacin (a respiratory quinolone) combined with cefoxitin was used instead. Since no bacteriological outcome was achieved, imipenem/cilastatin sodium was used instead; however, the result was still poor.

The possible causes of suboptimal antimicrobial therapy may include: history of COPD; poor underlying lung condition; ongoing bilateral closed-chest drainage; parts of chest wall activities are inhibited by chest tube; scratching of the pleura by the drainage tubes due to cough; severe pain restricts the drainage of the sputum, which subsequently results in lung infection. Empiric antibiotic use should be adjusted according to the epidemiological trend in hospitalacquired pneumonia in the ward. Patient's with hospital acquired pneumonia secondary to COPD are at high risk of Pseudomonas aeruginosa infection, for whom levofloxacin combined with piperacillin/tazobactam may be a good choice.

The patient has suffered from compression of the lung tissue due to pneumothorax. Blood gas analysis indicated hypoxia and carbon dioxide retention. The presence of a closed drainage tube in the chest had led to the limited mobility of the chest wall. In addition, poor drainage of sputum, poorly controlled pulmonary infection, and a prolonged disease course could easily induce infections with multi-drug resistant bacteria. After 1 month of closed-chest drainage, the effectiveness was poor and the pneumothorax was not absorbed, along with massive air accumulation in the subcutaneous tissues. Therefore, further surgical treatment is recommended.

After the surgery, the patient should be transferred to ICU for close monitoring. Special attention should be given to the blood gas analysis results, chest $\mathrm{X}$-ray findings, and vital signs. Since the patient had a history of COPD, oxygen inhalation therapy with high-concentration oxygen may lead to carbon dioxide retention. Thus, high-flow ventilation within a short period of time may be considered. Postoperative active drainage of sputum should be performed. Sputum culture and drug susceptibility testing should be performed repeatedly to identify the pathogen and thus inform antimicrobial use.

\section{Inputs from department of thoracic surgery}

Dr. Xuefeng Leng (from the Affiliated Hospital of Chengdu University): The patient was mainly presented with the rupture of bilateral pulmonary bulla after COPD, and the lung leak was obvious. After a long period of drainage and conservative treatment, the therapeutic effectiveness was poor. Massive air accumulation in subcutaneous and soft tissues is still present. Surgical treatment may be considered if there is no absolute surgical contraindication after an adequate anesthesiologic evaluation.

If the patient refuses surgery, pleural adhesion agent may be injected into the left side of the thoracic cavity, which generally can achieve satisfactory effectiveness. On the right side, injection of pleural adhesion agent may not be effective due to inadequate lung recruitment. Antimicrobial treatment may be applied firstly, followed by expectoration (aspiration of sputum by bronchoscope, if necessary). Negative pressure suction may be utilized if appropriate. 
After the lungs are recruited, a pleural adhesion agent is injected for treatment. In our center, three patients with severe grade III air leak failed to achieve effective pulmonary recruitment after negative pressure suction and other related treatments. Fortunately, the ruptured bullae were ultimately closed after repeated injection of pleural adhesion agents. The duration of conservative treatment can be long (may last 7-15 days). Furthermore, the feasibility of placing an intracavity valve in the target segment of the bronchus may be assessed in the endoscopy center.

If surgical treatment is planned, it is recommended to inject a pleural adhesion agent into the left thoracic cavity for conservative treatment. After the air leak disappears on the left side, resection of the right pulmonary bullae and pleurodesis can be performed. There were numerous similar patients in the department of respiratory medicine of our center. Examinations of this patient showed that the left pulmonary tissue was well recruited, whereas there were still large residual cavities on the right side. Massive air leak still existed on the right side after conservative treatment, along with poor lung recruitment, large residual cavities, and poor effectiveness of drainage alone. The massive air accumulation in subcutaneous and soft tissues may be due to the air leak at the right side.

Anesthesia may be associated with high risk due to the underlying COPD, poor pulmonary function, and poor tolerance to one-lung ventilation. Resection of the right lung bullae may be associated with relatively controllable surgical time and risk. Non-intubated anesthesia or singlelumen endotracheal intubation with low tidal volume can be considered. If the patient could tolerate intraoperatively, single-lumen endotracheal intubation for intraluminal blockage may be applied to maintain a clear surgical field and improve the operability. However, if the patient could not tolerate it, intermittent two lung ventilation can be a choice. Meanwhile, the right chest tube should be maintained to avoid the aggravation of pneumothorax caused by rupture of bullae.

Even if the patient underwent surgery, there would still be a risk of air leak at the wound surface due to the poor lung quality. After the surgery, in addition to the routine respiratory tract management and enhanced recovery after surgery (ERAS) in the department of thoracic surgery, active antimicrobial therapy and adequate drainage should be applied. If the duration of air leak at the operated side exceeds 5 days, a pleural adhesion agent can still be injected into the thoracic cavity to speed up wound healing. The chest tube can be withdrawn when the chest tube becomes free of air leak.

Dr. Qiang Lu (from Tangdu Hospital, the Fourth Military Medical University): According to the patient's current condition, surgery should be performed as soon as possible. Double-lumen endotracheal intubation is required during the surgery. During the anesthesia, the left and right main bronchi must be fully separated to improve the anesthetic safety and facilitate surgical maneuvers. A two-phased surgery may be the optimal option. The right side should be operated on firstly by using a video-assisted thoracoscopic (VATS) minimally invasive technique, during which a pad is inserted into the stitch nails to reduce postoperative air leak. Adequate lung recruitment should be achieved after surgery to eliminate residual cavity. Meanwhile, airway clearance management should be enhanced to facilitate expectoration. Any change in the general condition and the right chest should be closely monitored.

If the patient refuses surgery, the chest tube should replaced at the $2 \mathrm{nd}$ rib to eliminate the residual cavity on the right chest and keep patent drainage. Meanwhile, symptomatic antimicrobial treatment should continue.

\section{Inputs from department of anesthesiology}

Dr. Chunguang Wang (from Baoding First Central Hospital): The patient must receive a surgery since he had a history of COPD and has developed spontaneous pneumothorax. Conservative treatment was ineffective. Even worse, the symptoms of pneumothorax have worsened, along with massive subcutaneous emphysema. Simultaneous resection of bilateral pulmonary bullae is recommended.

The surgery should be performed under general anesthesia, with double lumen endotracheal intubation during one-lung ventilation. Tips for the anesthesia include: (I) use the closed-chest drainage tubes as large as possible and maintain the drainage tubes unobstructed, so that the air in the chest cavity can be thoroughly removed; (II) induction of anesthesia should be as stable as possible, so as to avoid increased intrathoracic pressure caused by cough or other factors; (III) pressure should be applied to control ventilation, so as to minimize the amount of massive air entering the chest cavity or subcutaneous tissues due to positive pressure ventilation; and (IV) use anticholinergic drugs to reduce the increase in respiratory secretions (which can increase airway pressure). If the patient refuses surgery, use the closed-chest drainage tubes as large as possible and maintain the drainage tubes unobstructed, so that the air in the chest cavity can be thoroughly removed. 


\section{Inputs from department of rehabilitation}

Dr. Fuanjuan Lei (from Tangdu Hospital): Surgical treatment is recommended. One month after the symptomatic conservative treatment, CT revealed the presence of pneumothorax on both sides of the chest, along with massive air accumulation in the subcutaneous tissue of the scrotums, severe atelectasis on the right side of the chest, low partial pressure of oxygen $\left(\mathrm{PaO}_{2}\right)$, and severe systemic symptoms. Conservative treatment can no longer improve atelectasis or hypoxia.

Since the patient is old and has severe conditions, he cannot tolerate surgery on both sides of the chest. Therefore, the surgery should be two-phased: on the right side first, and on the left, subsequently. The surgery should be performed under general anesthesia, during which arterial blood gas variables, heart rate, and blood pressure should be closely monitored. Antimicrobial therapy and nutritional support should always be offered no matter whether the patient agrees to receive a surgery. Physical therapists may guide the patient to receive training on breathing and sputum clearance and thus promote their recovery.

\section{Discussion and summary}

Pneumothorax is often unilateral and only $1 \%$ of patients may develop bilateral pneumothorax. When pneumothorax occurs, the patients often suffer from a sudden onset of difficulty in breathing along with chest pain; however, it may also be asymptomatic in a small proportion of patients, and the condition is identified only during a health checkup $(1,2)$. Bilateral pneumothorax is often seen in old patients with underlying chronic lung diseases. As a sudden and complex condition, it can easily cause respiratory failure and yield high mortality. Timely diagnose and effectively closed-chest drainage are crucial for avoiding/controlling respiratory failure caused by bilateral pneumothorax (3).

For the subsequent treatment of our current case, surgical treatment is strongly recommended by expert groups (Thoracic Surgeons, Anesthesiologists, Pulmonologists and rehabilitators). Surgery should be done under general anesthesia using double-lumen endotracheal intubation, which not only can ensure the depth of anesthesia but also maintains a clear operative field and facilitates surgical maneuvers. Considering the poor general condition, poor lung function, and lung infections in the patient, some experts also recommend non-intubated anesthesia under spontaneous breathing or single-lumen endotracheal tube anesthesia. By using sedative and analgesic drugs with rapid onset, short half-life, and little impact on circulation and respiration, non-intubated anesthesia can maximize the physiological stability during surgery and promote postoperative recovery (4). Patients who are expected to have difficulty in withdrawing ventilatory support (e.g., patients with COPD, interstitial lung disease, and/ or poor pulmonary function) will benefit more from this technique (5). Patient's complete collaboration beforeduring surgery is paramount, and should be taken in to account when such approach is chosen.

Interestingly, there were different opinions on whether the surgery should be performed on one side or both sides or where a one-phase or two-phased surgery should be performed. An expert from the department of anesthesiology suggested a surgery on both sides in one single phase, whereas experts from other departments agreed that a twophased surgery should be adopted based on the following considerations: (I) the patient is old, with a long and severe disease history; (II) the patient cannot tolerate an operation on both sides within one single phase; (III) the pneumothorax was large on the right side, and an operation on the right side before on the left side is more feasible. An expert from the department of thoracic surgery also advised the injection of adhesion agent into the left thoracic cavity to treat pneumothorax. A variety of adhesion agents including talcum powder suspension, high-concentration glucose, autologous venous blood, human serum albumin, erythromycin, blood coagulation enzymes, and diluted iodophor have been described. However, their safety and effectiveness still need to be further elucidated in studies with large sample sizes (6).

Experts from most disciplines recommended that the patient should be transferred to ICU after surgery, focusing on respiratory tract management, antimicrobial therapy, drainage tube management, nutritional support, and rehabilitation exercises. After multidisciplinary consultations, we decided to perform two-staged surgical treatment under general anesthesia with double-lumen tracheal intubation for the patient. The VATS resection of right pulmonary bullae, suturing, and fixation of pleura was performed firstly, and then the patient was transferred to ICU.

\section{Treatment}

After 1 month of conservative treatment, the patient underwent VATS resection of right pulmonary bullae, suturing, and fixation of pleura under general anesthesia 
with double-lumen tracheal intubation. After the surgery, while there was no obvious air leak on the right chest tube, air leak persisted on the left chest tube. A second chest CT indicated that the lungs were well recruited and the subcutaneous emphysema gradually subsided (Figure 3). One week later, VATS resection of left pulmonary bullae, suturing, and fixation of pleura was performed. After the surgery, air leak did no longer exist on the left chest tube. Seven days after surgery, both lungs were well recruited and the patient was discharged from the hospital (Figure 4).

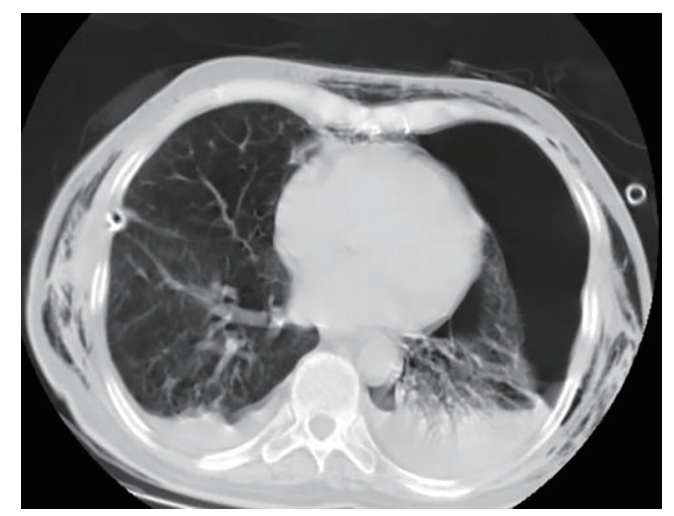

Figure 3 CT findings after the operation on the right side of the chest: Pneumothorax persisted on the left side. After the right side was operated (with drainage tube indwelling), there were just a little amount of pleural effusions on both sides, along with segmental atelectasis at the left lower lung. The air accumulation in the subcutaneous tissue of the chest wall significantly decreased. CT, computed tomography.

\section{Conclusions}

The MDT, mainly for tumor diagnosis and treatment, has been widely recognized. It integrates the opinions of experts from various disciplines and provides one-stop service for the patients. It is also helpful to provide more comprehensive and reasonable treatment decisions for the diagnosis and treatment of critically ill patients (7). In our current case, based on the inputs of an MDT composed of experts from the department of thoracic surgery, ICU, department of respiratory medicine, department of anesthesiology, and department of rehabilitation, two-staged VATS resection of pulmonary bullae, suturing, and fixation of pleura was performed. After the surgery, the patient was transferred to ICU, where he received antimicrobial therapy, nutritional support, and rehabilitation exercises. The patient recovered well within one week after the surgery. MDT can help health care providers to make the right decisions at the critical time point in the treatment of disease, allowing patients to receive appropriate treatment and maximizing patient safety and quality of care.

\section{Expert opinion 1: thoracic surgery under general anesthesia (Dr. Kurt Ruetzler)}

As an anesthesiologist, I would definitely prefer thoracic surgery under general anesthesia including tracheal intubation.

Bilateral surgery might be possible, but mostly depends on pulmonary function of both lungs. Patient has a long history of COPD, therefore pulmonary function might be
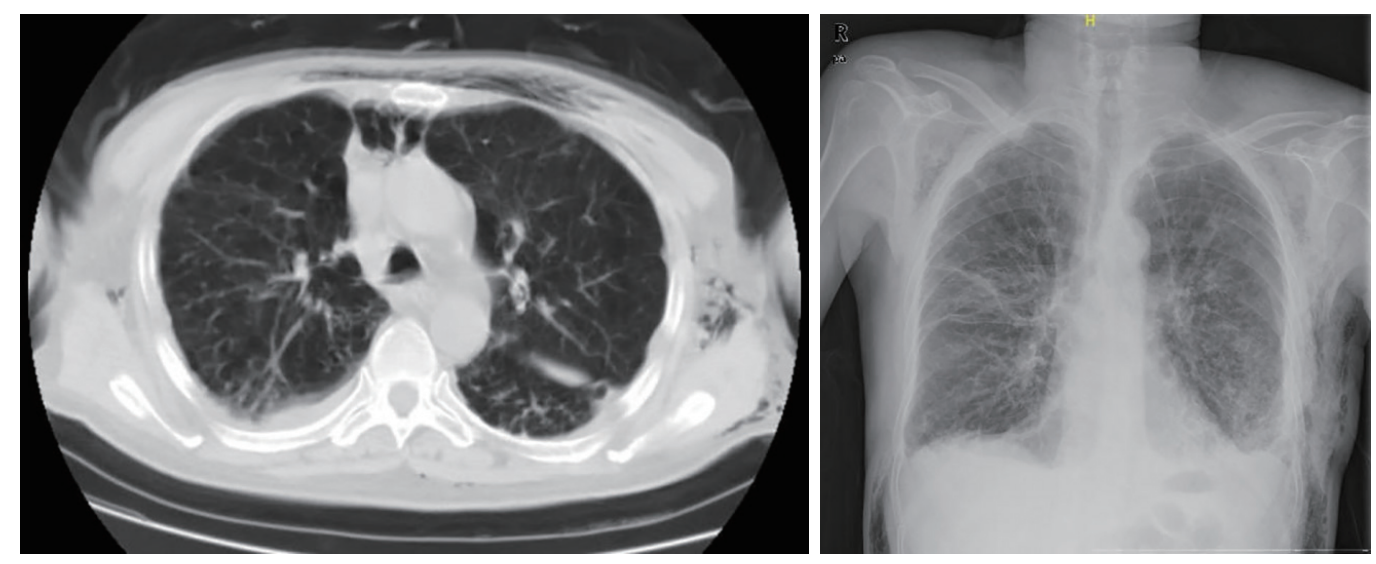

Figure 4 CT findings after the operation on both sides of the chest: both pneumothorax and subcutaneously accumulated air were absorbed. CT, computed tomography. 
decreased, but bilateral surgery might still be an option.

I would suggest intravenous general anesthesia. Intubation can be done using a double-lumen tube (DLT); although a single-lumen tube in combination with a bronchial blocker might be an appropriate option. The patient most likely needs prolonged ventilatory support on ICU and using a single-lumen tube during surgery prevents from changing the DLT to the obligate singlelumen tube for ICU. First, surgery should be performed on the right side, followed by the left. During single-lung ventilation $5 \mathrm{mmHg}$ of PEEP and $2-4 \mathrm{~L} / \mathrm{min} 100 \%$ oxygen should be applied to the collapsed lung in order to improve (apneic) oxygenation. Positive pressure ventilation during single-lung ventilation might be tricky, but limiting the peak pressure and the tidal volume to about 300-320 cc, and adjusting the respiration rate might be the key points. Continuous muscle relaxation might be helpful. PEEP to the ventilated lungs should be reduced as much as possible.

\section{Expert opinion 2: conservative therapy should be earlier? (Dr. Hiran C. Fernando)}

I think the patient should have been considered for surgery at a much early time point (not at 1 month as reported here). I don't understand why there was a delay in making the decision to operate. I do not perform non-intubated surgery, so it is hard for me to comment definitively. However for those centers that perform non-intubated surgery there may be advantages when operating on such a patient using this approach. Positive pressure ventilation may lead to a greater air-leak in the ventilated lung if a standard double lumen-tube endotracheal intubation was used. On the other hand, this would be the best way to have a secure airway during the procedure.

I would plan on performing both sides the same day. I would start with the side with the worst air-leak. I would use standard double lumen endotracheal intubation. Chesttubes would be kept in place bilaterally. I would prep the patient's chest with the chest-tube in place and remove this after stopping ventilation to the operative lung. I would keep the chest-tube in the ventilated (down) lung on waterseal, to minimize the air-leak during positive pressure ventilation. After completing one side, if the patient has been stable, I would then reposition the patient and operate on the opposite lung.

This is nice discussion on a challenging clinical case. The only other recommendation I would make or question is how long should conservative therapy be trialed. A month seems like a long time- although this was ultimately successful in this patient. I would have intervened sooner, unless I felt the patient could be medically optimized by delaying.

\section{Expert opinion 3: bilateral intervention during one general anesthetic is safe (Drs. Sebastien Gilbert \& Ching Yeung)}

This patient had risk factors for spontaneous bilateral primary spontaneous pneumothorax: the presence of underlying lung disease (COPD), low BMI [16], and the presence of blebs and bullae.

Given the initial diagnosis of tension pneumothorax, we agree with the initial chest tube placements. Normally for tension pneumothorax, we would recommend intervention beyond simple pleural drainage given the potential lifethreatening nature of this problem should it recur in a setting of limited access to rapid health care. This case is interesting as the patient's parenchymal disease and low BMI puts him at increased risk of post-op complications and prolonged air leak.

Although temporary decompression of a region of subcutaneous emphysema may be achieved by incising the skin and soft tissues (i.e., blow holes), our preferred management strategy is ensuring adequate drainage of the air leak using multiple chest drains on continuous suction. Subcutaneous emphysema is typically of no other consequence than temporary alteration in cosmesis. Blow holes do not decrease the need for additional chest drains to control an air leak, may get infected, cause additional pain and suffering, and permanently alter cosmesis.

The determination of surgery versus conservative management at the time of initial diagnosis would take into consideration whether potentially resectable areas of bullous emphysema or pulmonary blebs are present on CT scan. Additionally, if there is no clearly identifiable culprit lesion but severe diffuse bullous emphysema, consideration should be given to surgical pleurodesis if the air leak resolves and the patient can tolerate anesthesia. In any case, the lung volume reduction surgery (LVRS) data tells us that bilateral intervention during one general anesthetic is safe.

If there were factors to preclude general anesthesia and surgical intervention, it would be reasonable, in our opinion, to perform bedside chemical pleurodesis.

Regarding the prolonged air leak, we recently established a predictive model to identify patients at risk for prolonged air leak after lung resection. These risk factors include male 
gender, smoking history, $\mathrm{BMI}<25$, medical research council (MRC) dyspnea score above 1, and diffusion capacity for carbon monoxide below $80 \%$. Assuming that this patient has poor pulmonary function testing (PFT) and poor MRC dyspnea score, according to our scoring system, this patient would have been at an increased risk for an air leak (8). We are happy to read that this patient did not develop a prolonged air leak after his VATS procedure.

For surgical approach, we have had good success with VATS bullectomy and pleurectomy. Our operations are performed under general anesthesia and double lumen endotracheal tube lung isolation. Blebs and bullae are resected with Endo GIA stapling devices. If there is a concern about prolonged air leak, we use felt reinforced staplers (Endo GIA Reinforced Reload with tri-staple technology). We conduct a partial parietal pleura pleurectomy.

Post-operatively, we use one or two chest tubes maintained on suction for 24-48 hours. Given that VATS bullectomy and pleurectomy is safe in bilateral pneumothoraces and that recurrent tension pneumothorax could pose a threat to the patient's life, we believe that surgical intervention was indicated this patient. In simple uncomplicated spontaneous pneumothorax, we only recommend surgical intervention following the first recurrence. Our institutional rate of recurrent pneumothorax after VATS bullectomy and pleurectomy is approximately $7 \%$ (9).

\section{Expert opinion 4: (Dr. Pier Luigi Filosso)}

I believe that this is a very interesting case very unusual in the routine clinical practice. The MDT adoption made possible a prompt and effective treatment of such rare disease.

\section{Acknowledgements}

Funding: This project was supported by the Zhejiang Provincial Natural Science Foundation of China (No. LQ18H160029) and Taizhou Postdoctoral Science
Foundation (No. 201702).

\section{Footnote}

Conflicts of Interest: The authors have no conflicts of interest to declare.

\section{References}

1. Sayar A, Turna A, Metin M, et al. Simultaneous bilateral spontaneous pneumothorax report of 12 cases and review of the literature. Acta Chir Belg 2004;104:572-6.

2. Lee SC, Cheng YL, Huang CW, et al. Simultaneous bilateral primary spontaneous pneumothorax. Respirology 2008;13:145-8.

3. Brown SG, Ball EL, Perrin K, et al. Study protocol for a randomised controlled trial of invasive versus conservative management of primary spontaneous pneumothorax. BMJ Open 2016;6:e011826.

4. Landoni G, Zangrillo A, Cabrini L. Noninvasive ventilation after cardiac and thoracic surgery in adult patients:a review.Journal of cardiothoracic and vascular anesthesia. J Cardiothorac Vasc Anesth 2012;26:917-22.

5. Guarracino F, Gemignani R, Pratesi G, et al. Awake palliative thoracic surgery in a high-risk patient:one-lung, non-invasive ventilation combined with epidural blockade. Anaesthesia 2008;63:761-3.

6. Ma H, Jia W, Li Y. Analyzing thoracic cavity gas during pneumothorax treatment to monitor the pneumothorax conditions in real time for updating the treatment strategy: A pilot study. Technology 2015;3:189.

7. Munshi A, Sengar M. Multidisciplinary care in oncology: Are we united? Acta Oncol 2011;50:314-6.

8. Gilbert S, Maghera S, Seely AJ, et al. Identifying patients at higher risk of prolonged air leak after lung resection. Ann Thorac Surg 2016;102:1674-9.

9. Addas RA, Shamji FM, Sundaresan SR, et al. Is VATS bullectomy and pleurectomy an effective method for the management of spontaneous pneumothorax? Open J Thorac Surg 2016;6:25-31.
Cite this article as: $\mathrm{Li} \mathrm{X,} \mathrm{Su} \mathrm{X,} \mathrm{Chen} \mathrm{B,} \mathrm{Yao} \mathrm{H,} \mathrm{Yu} \mathrm{Y,} \mathrm{Leng}$ X, Lu Q, Wang C, Lei J, Ruetzler K, Fernando HC, Gilbert S, Ching Y, Filosso PL, Shen J, Zhu C; Written on behalf of AME Thoracic Surgery Collaborative Group. Multidisciplinary team approach on a case of bilateral tension pneumothorax. J Thorac Dis 2018;10(4):2528-2536. doi: 10.21037/jtd.2018.04.81 